Internat. J. Math. \& Math. Sci.

Vol. 23, No. 3 (2000) 189-195

S0161171200002520

(C) Hindawi Publishing Corp.

\title{
THE $\ell$-TRANSLATIVITY OF ABEL-TYPE MATRIX
}

\author{
MULATU LEMMA
}

(Received 7 December 1998)

ABSTRACT. Lemma introduced the Abel-type matrix $A_{\alpha, t}$ defined by $a_{n k}=\left(\begin{array}{c}k+\alpha \\ k\end{array}\right) t_{n}^{k+1}(1-$ $\left.t_{n}\right)^{\alpha+1}$, where $\alpha>-1,0<t_{n}<1$, for all $n$, and $\lim t_{n}=1$; and studied it as mappings into $\ell$. In this paper, we extend our study of this matrix and investigate its translativity in the $\ell-\ell$ setting.

Keywords and phrases. $\ell-\ell$ method, $\ell$-translative, Abel-type power series method, Abeltype matrix.

2000 Mathematics Subject Classification. Primary 40A05, 40D99; Secondary 40 C05.

1. Background. In [1], Borwein proved that the Abel-type power series method of summability denoted by $A_{\alpha}(\alpha>-1)$, is translative in the ordinary summability $(c-c)$ setting. So, it natural to ask if there is a theory in the $\ell-\ell$ setting that parallel the theory of $A_{\alpha}$ in the $c$ - $c$ setting. The answer is affirmative, and have provided the present study.

2. Basic notation and definitions. Let $A=\left(a_{n k}\right)$ be an infinite matrix defining a sequence to a sequence summability transformation given by

$$
(A x)_{n}=\sum_{k=0}^{\infty} a_{n k} x_{k},
$$

where $(A x)_{n}$ denotes the $n$th term of the image sequence $A x$. Let $y$ be a complex number sequence. Throughout this paper, we use the following basic notations and definitions:

(i) $c=\{$ the set of all convergent complex number seqences $\}$,

(ii) $\ell=\left\{y: \sum_{k=0}^{\infty}\left|y_{k}\right|\right.$ converges $\}$,

(iii) $\ell(A)=\{y: A y \in \ell\}$,

(iv) $c(A)=\{y: y$ is summable by $\mathrm{A}\}$.

DEFINITION 1. If $X$ and $Y$ are sets of complex number sequences, then the matrix $A$ is called an $X-Y$ matrix if the image $A u$ of $u$ under the transformation $A$ is in $Y$ whenever $u$ is in $X$.

DEFinItion 2. The summability matrix $A$ is said to be $\ell$-translative for the sequence $u$ in $\ell(A)$ provided that each of the sequences $T_{u}$ and $S_{u}$ is in $\ell(A)$, where $T_{u}=\left\{u_{1}, u_{2}, u_{3}, \ldots\right\}$ and $S_{u}=\left\{0, u_{0}, u_{1}, \ldots\right\}$. 


\section{The main results}

Proposition 1. Every $\ell-\ell A_{\alpha, t}$ matrix is $\ell$-translative for each sequence $x \in \ell$.

THEOREM 1. Every $\ell-\ell A_{\alpha, t}$ matrix is $\ell$-translative for those sequences $x \in \ell\left(A_{\alpha, t}\right)$ for which $\left\{x_{k} / k\right\} \in \ell, k=1,2,3, \ldots$.

Proof. Suppose that $x$ is a sequence in $\ell\left(A_{\alpha, t}\right)$ for which $\left\{x_{k} / k\right\} \in \ell$. We show that

(1) $T_{x} \in \ell\left(A_{\alpha, t}\right)$, and

(2) $S_{x} \in \ell\left(A_{\alpha, l}\right)$, where $T_{x}$ and $S_{x}$ are as defined in Definition 2. Let us first show that (1) holds.

Note that

$$
\begin{aligned}
\left|\left(A_{\alpha, t} T_{x}\right)_{n}\right| & =\left(1-t_{n}\right)^{\alpha+1}\left|\sum_{k=0}^{\infty}\left(\begin{array}{c}
k+\alpha \\
k
\end{array}\right) x_{k+1} t_{n}^{k}\right| \\
& =\frac{\left(1-t_{n}\right)^{\alpha+1}}{t_{n}}\left|\sum_{k=0}^{\infty}\left(\begin{array}{c}
k+\alpha \\
k
\end{array}\right) x_{k+1} t_{n}^{k+1}\right| \\
& =\frac{\left(1-t_{n}\right)^{\alpha+1}}{t_{n}}\left|\sum_{k=1}^{\infty}\left(\begin{array}{c}
k-1+\alpha \\
k-1
\end{array}\right) x_{k} t_{n}^{k}\right| \\
& =\frac{\left(1-t_{n}\right)^{\alpha+1}}{t_{n}}\left|\sum_{k=1}^{\infty}\left(\begin{array}{c}
k+\alpha \\
k
\end{array}\right) x_{k} t_{n}^{k} \frac{k}{k+\alpha}\right| \\
& =\frac{\left(1-t_{n}\right)^{\alpha+1}}{t_{n}}\left|\sum_{k=1}^{\infty}\left(\begin{array}{c}
k+\alpha \\
k
\end{array}\right) x_{k} t_{n}^{k}\left(1-\frac{\alpha}{k+\alpha}\right)\right| \\
& \leq A_{n}+B_{n},
\end{aligned}
$$

where

$$
A_{n}=\frac{\left(1-t_{n}\right)^{\alpha+1}}{t_{n}}\left|\sum_{k=1}^{\infty}\left(\begin{array}{c}
k+\alpha \\
k
\end{array}\right) x_{k} t_{n}^{k}\right|
$$

and

$$
B_{n}=\frac{|\alpha|\left(1-t_{n}\right)^{\alpha+1}}{t_{n}}\left|\sum_{k=1}^{\alpha}\left(\begin{array}{c}
k+\alpha \\
k
\end{array}\right) \frac{x_{k}}{k+\alpha} t_{n}^{k}\right| .
$$

The use of the triangle inequality in equation (3.1) is legitimate as the radii of convergence of the two power series are at least 1 . Now if we show that both $A$ and $B$ are in $\ell$, then (1) holds. But the conditions that $A \in \ell$ and $B \in \ell$ follow easily from the hypotheses that $x \in \ell\left(A_{\alpha, t}\right)$ and $\left\{x_{k} / k\right\} \in \ell$, respectively. Next, we show that (2) holds as follows. We have

$$
\begin{aligned}
\left|\left(A_{\alpha, t} S_{x}\right)_{n}\right| & =\left(1-t_{n}\right)^{\alpha+1}\left|\sum_{k=1}^{\infty}\left(\begin{array}{c}
k+\alpha \\
k
\end{array}\right) x_{k-1} t_{n}^{k}\right| \\
& =\left(1-t_{n}\right)^{\alpha+1}\left|\sum_{k=0}^{\infty}\left(\begin{array}{c}
k+\alpha+1 \\
k+1
\end{array}\right) x_{k} t_{n}^{k+1}\right|
\end{aligned}
$$




$$
\begin{aligned}
& =\left(1-t_{n}\right)^{\alpha+1}\left|\sum_{k=0}^{\infty}\left(\begin{array}{c}
k+\alpha \\
k
\end{array}\right) x_{k} t_{n}^{k+1}\left(\frac{k+\alpha+1}{k+1}\right)\right| \\
& =\left(1-t_{n}\right)^{\alpha+1}\left|\sum_{k=0}^{\infty}\left(\begin{array}{c}
k+\alpha \\
k
\end{array}\right) x_{k} t_{n}^{k+1}\left(1+\frac{\alpha}{k+1}\right)\right| \\
& \leq E_{n}+F_{n},
\end{aligned}
$$

where

$$
E_{n}=\left(1-t_{n}\right)^{\alpha+1}\left|\sum_{k=0}^{\infty}\left(\begin{array}{c}
k+\alpha \\
k
\end{array}\right) x_{k} t_{n}^{k}\right|
$$

and

$$
F_{n}=\left(1-t_{n}\right)^{\alpha+1}|\alpha|\left|\sum_{k=0}^{\infty}\left(\begin{array}{c}
k+\alpha \\
k
\end{array}\right) \frac{x_{k}}{k+1} t_{n}^{k+1}\right| .
$$

The use of the triangle inequality in (3.4) is justified as above. If we show that $E$ and $F$ are in $\ell$, then (2) holds. But the hypothesis that $x \in \ell\left(A_{\alpha, t}\right)$ and $\left\{x_{k} / k\right\} \in \ell$ implies that both $E$ and $F$ are in $\ell$, respectively, and hence the theorem follows.

Here, we remark that a sequence $x$ defined by $x_{k}=(-1)^{k} / k$ is one of the sequences which satisfies the condition of Theorem 1 .

THEOREM 2. Suppose that $-1<\alpha \leq 0$, then every $\ell-\ell A_{\alpha, t}$ matrix is $\ell$-traslative for each $A_{\alpha}$-summable sequence $x$ in $\ell\left(A_{\alpha, t}\right)$.

Proof. Let $x \in c\left(A_{\alpha}\right) \cap \ell\left(A_{\alpha, t}\right)$. We show that

(1) $T_{x} \in \ell\left(A_{\alpha, t}\right)$,

(2) $S_{x} \in \ell\left(A_{\alpha, t}\right)$.

First, let us show that (1) holds. Since the case $\alpha=0$ was already proved by J. Fridy in [2], here we only consider the case $-1<\alpha<0$. Note that

$$
\begin{aligned}
\left|\left(A_{\alpha, t} T_{x}\right)_{n}\right| & =\left(1-t_{n}\right)^{\alpha+1}\left|\sum_{k=0}^{\infty}\left(\begin{array}{c}
k+\alpha \\
k
\end{array}\right) x_{k+1} t_{n}^{k}\right| \\
& =\frac{\left(1-t_{n}\right)^{\alpha+1}}{t_{n}}\left|\sum_{k=0}^{\infty}\left(\begin{array}{c}
k+\alpha \\
k
\end{array}\right) x_{k+1} t_{n}^{k+1}\right| \\
& =\frac{\left(1-t_{n}\right)^{\alpha+1}}{t_{n}}\left|\sum_{k=1}^{\infty}\left(\begin{array}{c}
k-1+\alpha \\
k-1
\end{array}\right) x_{k} t_{n}^{k}\right| \\
& =\frac{\left(1-t_{n}\right)^{\alpha+1}}{t_{n}}\left|\sum_{k=1}^{\infty}\left(\begin{array}{c}
k+\alpha \\
k
\end{array}\right) x_{k} t_{n}^{k} \frac{k}{k+\alpha}\right| \\
& =\frac{\left(1-t_{n}\right)^{\alpha+1}}{t_{n}}\left|\sum_{k=1}^{\infty}\left(\begin{array}{c}
k+\alpha \\
k
\end{array}\right) x_{k} t_{n}^{k}\left(1-\frac{\alpha}{k+\alpha}\right)\right| \\
& \leq A_{n}+B_{n},
\end{aligned}
$$

where

$$
A_{n}=\left(1-t_{n}\right)^{\alpha+1}\left|\sum_{k=1}^{\infty}\left(\begin{array}{c}
k+\alpha \\
k
\end{array}\right) x_{k} t_{n}^{k}\right|
$$


and

$$
B_{n}=-\alpha\left(1-t_{n}\right)^{\alpha+1}\left|\sum_{k=1}^{\infty}\left(\begin{array}{c}
k+\alpha \\
k
\end{array}\right) t_{n}^{k} \frac{x_{k}}{k+\alpha}\right| .
$$

Now if we show that both $A$ and $B$ are in $\ell$, then (1) holds. But the condition that $A \in \ell$ follows from the hypothesis that $x \in \ell\left(A_{\alpha, t}\right)$ and $B \in \ell$ are shown as follows. Observe that

$$
B_{n}<\left(1-t_{n}\right)^{\alpha+1}\left|x_{1}\right|+\left(1-t_{n}\right)^{\alpha+1}\left|\sum_{k=2}^{\infty}\left(\begin{array}{c}
k+\alpha \\
k
\end{array}\right) t_{n}^{k} \frac{x_{k}}{k+\alpha}\right|=C_{n}+D_{n},
$$

where

$$
C_{n}=\left|x_{1}\right|\left(1-t_{n}\right)^{\alpha+1}
$$

and

$$
D_{n}=\left(1-t_{n}\right)^{\alpha+1}\left|\sum_{k=2}^{\infty}\left(\begin{array}{c}
k+\alpha \\
k
\end{array}\right) t_{n}^{k} \frac{x_{k}}{k+\alpha}\right| .
$$

By [3, Thm. 1], the hypothesis that $A_{\alpha, t} \ell-\ell$ implies that $C \in \ell$, hence there remains only to show $D \in \ell$ to show that $B \in \ell$. Observe that

$$
\begin{aligned}
D_{n} & =\frac{\left(1-t_{n}\right)^{\alpha+1}}{t_{n}^{\alpha}}\left|\sum_{k=2}^{\infty}\left(\begin{array}{c}
k+\alpha \\
k
\end{array}\right) x_{k}\left(\int_{0}^{t_{n}} t^{k+\alpha-1} d t\right)\right| \\
& =\frac{\left(1-t_{n}\right)^{\alpha+1}}{t_{n}^{\alpha}}\left|\int_{0}^{t_{n}} d t\left(\sum_{k=2}^{\infty}\left(\begin{array}{c}
k+\alpha \\
k
\end{array}\right) x_{k} t^{k+\alpha-1}\right)\right| .
\end{aligned}
$$

The interchanging of the integral and the summation is legitimate as the radius of convergence of the power series

$$
\sum_{k=2}^{\infty}\left(\begin{array}{c}
k+\alpha \\
k
\end{array}\right) x_{k} t^{k+\alpha-1}
$$

is at least 1 by [3, Prop. 1], and hence the power series converges absolutely and uniformly for $0 \leq t \leq t_{n}$. Now we let

$$
F(t)=\sum_{k=2}^{\infty}\left(\begin{array}{c}
k+\alpha \\
k
\end{array}\right) x_{k} t^{k+\alpha-1}
$$

Then, we have

$$
F(t)(1-t)^{\alpha+1}=(1-t)^{\alpha+1} \sum_{k=2}^{\infty}\left(\begin{array}{c}
k+\alpha \\
k
\end{array}\right) x_{k} t^{k+\alpha-1}
$$

and the hypothesis that $x \in c\left(A_{\alpha}\right)$ implies that

$$
\lim _{t \rightarrow 1^{-}} F(t)(1-t)^{\alpha+1}=A \text { (finite) for } 0<t<1 .
$$


We also have

$$
\lim _{t \rightarrow 0} F(t)(1-t)^{\alpha+1}=0 .
$$

Now equations (3.17) and (3.18) yield that

$$
\left|F(t)(1-t)^{\alpha+1}\right| \leq M_{1} \quad \text { for some } M_{1}>0
$$

and hence

$$
|F(t)| \leq M_{1}(1-t)^{-(\alpha+1)}
$$

So, we have

$$
\begin{aligned}
D_{n} & =\frac{\left(1-t_{n}\right)^{\alpha+1}}{t_{n}^{\alpha}}\left|\int_{0}^{t_{n}} F(t) d t\right| \\
& \leq M_{2}\left(1-t_{n}\right)^{\alpha+1} \int_{0}^{t_{n}}|F(t)| d t \quad \text { for some } M_{2}>0 \\
& \leq M_{1} M_{2}\left(1-t_{n}\right)^{\alpha+1} \int_{0}^{t_{n}}(1-t)^{-(\alpha+1)} d t \\
& =\frac{M_{1} M_{2}}{\alpha}\left(1-t_{n}\right)-\frac{M_{1} M_{2}}{\alpha}\left(1-t_{n}\right)^{\alpha+1} \\
& \leq-\frac{2 M_{1} M_{2}}{\alpha}\left(1-t_{n}\right)^{\alpha+1}
\end{aligned}
$$

By [3, Thm. 1], the hypothesis that $A_{\alpha, t} \ell-\ell$ implies that $(1-t)^{\alpha+1} \in \ell$, and hence $D \in \ell$. Next we show that (2) holds. We have

$$
\begin{aligned}
\left|\left(A_{\alpha, t} S_{x}\right)_{n}\right| & =\left(1-t_{n}\right)^{\alpha+1}\left|\sum_{k=1}^{\infty}\left(\begin{array}{c}
k+\alpha \\
k
\end{array}\right) x_{k-1} t_{n}^{k}\right| \\
& =\left(1-t_{n}\right)^{\alpha+1}\left|\sum_{k=0}^{\infty}\left(\begin{array}{c}
k+\alpha+1 \\
k+1
\end{array}\right) x_{k} t_{n}^{k+1}\right| \\
& =\left(1-t_{n}\right)^{\alpha+1}\left|\sum_{k=0}^{\infty}\left(\begin{array}{c}
k+\alpha \\
k
\end{array}\right) x_{k} t_{n}^{k+1}\left(\frac{k+\alpha+1}{k+1}\right)\right| \\
& =\left(1-t_{n}\right)^{\alpha+1}\left|\sum_{k=0}^{\infty}\left(\begin{array}{c}
k+\alpha \\
k
\end{array}\right) x_{k} t_{n}^{k+1}\left(1+\frac{\alpha}{k+1}\right)\right| \\
& \leq E_{n}+F_{n},
\end{aligned}
$$

where

$$
E_{n}=\left(1-t_{n}\right)^{\alpha+1}\left|\sum_{k=0}^{\infty}\left(\begin{array}{c}
k+\alpha \\
k
\end{array}\right) x_{k} t_{n}^{k}\right|
$$

and

$$
F_{n}=-\left(1-t_{n}\right)^{\alpha+1} \alpha\left|\sum_{k=0}^{\infty}\left(\begin{array}{c}
k+\alpha \\
k
\end{array}\right) \frac{x_{k}}{k+1} t_{n}^{k+1}\right| .
$$

Now if we show that both $E$ and $F$ are in $\ell$, then (2) follows. But the hypothesis that $x \in \ell\left(A_{\alpha, t}\right)$ implies that $E \in \ell$, and $F \in \ell$ follows using the same technique used in showing $D \in \ell$ in (1) in the proof of Theorem 2. Hence the theorem is proved. 
COROLlary 1. Suppose that $-1 \leq \alpha \leq 0$; then every $\ell-\ell A_{\alpha, t}$ matrix is $\ell$-translative for the class of all sequence $x$ whose partial sum is bounded.

Proof. By [3, Thm. 8], $x$ is in $\ell\left(A_{\alpha, t}\right)$ and it is easy to see that $x$ is also in $c\left(A_{\alpha}\right)$. Hence the assertion follows by Theorem 2 .

COROLLARY 2. Suppose that $-1 \leq \alpha \leq 0$; then every $\ell-\ell A_{\alpha, t}$ matrix is $\ell$-translative for the unbounded sequence $x$ defined by

$$
x_{k}=(-1)^{k} \frac{k+\alpha+1}{\alpha+1} .
$$

Proof. Since $x \in \mathcal{c}\left(A_{\alpha}\right) \cap \ell\left(A_{\alpha, t}\right)$, the corollary easily follows by Theorem 2 .

THEOrem 3. Suppose that $\alpha>0$ and $(1-t) \in \ell$; then every $\ell-\ell A_{\alpha, t}$ matrix is $\ell$ translative for each $A_{\alpha}$-summable sequence in $\ell\left(A_{\alpha, t}\right)$.

Proof. Suppose that $x \in c\left(A_{\alpha}\right) \cap \ell\left(A_{\alpha, t}\right)$. To prove the theorem, we need to show that both $A_{\alpha, t} T_{x}$ and $A_{\alpha, t} S_{x}$ are in $\ell$. We have

$$
\left|\left(A_{\alpha, t} T_{x}\right)_{n}\right|=\left(1-t_{n}\right)^{\alpha+1}\left|\sum_{k=0}^{\infty}\left(\begin{array}{c}
k+\alpha \\
k
\end{array}\right) x_{k+1} t_{n}^{k}\right|,
$$

and by referring to the proof of Theorem 1, we can express $\left|\left(A_{\alpha, t} T_{x}\right)_{n}\right|$ as

$$
\left|\left(A_{\alpha, t} T_{x}\right)_{n}\right| \leq A_{n}+B_{n}
$$

where

$$
A_{n}=\frac{\left(1-t_{n}\right)^{\alpha+1}}{t_{n}}\left|\sum_{k=1}^{\infty}\left(\begin{array}{c}
k+\alpha \\
k
\end{array}\right) x_{k} t_{n}^{k}\right|
$$

and

$$
B_{n}=\frac{\alpha\left(1-t_{n}\right)^{\alpha+1}}{t_{n}}\left|\sum_{k=1}^{\infty}\left(\begin{array}{c}
k+\alpha \\
k
\end{array}\right) \frac{x_{k}}{k+\alpha} t_{n}^{k}\right| .
$$

Now if we show that both $A$ and $B$ are in $\ell$, then $A_{\alpha, t} T_{x}$ is in $\ell$. The condition that $A \in \ell$ follows easily since $A_{\alpha, t}$ is an $\ell-\ell$ matrix and $x \in \ell\left(A_{\alpha, t}\right)$. The condition that $B \in \ell$ is shown as follows. Using the triangle inequality, we have

$$
B_{n} \leq C_{n}+D_{n}
$$

where

$$
C_{n}=\alpha\left|x_{1}\right|\left(1-t_{n}\right)^{\alpha+1}
$$

and

$$
D_{n}=\alpha\left(1-t_{n}\right)^{\alpha+1}\left|\sum_{k=2}^{\infty}\left(\begin{array}{c}
k+\alpha \\
k
\end{array}\right) t_{n}^{k} \frac{x_{k}}{k+\alpha}\right| .
$$


We have $B \in \ell$, if we show that both $C$ and $D$ are in $\ell$. The condition that $C \in \ell$ follows easily as $(1-t)^{\alpha+1} \in \ell$ by [3, Thm. 1], and $D \in \ell$ can be shown as follows. Note that following exactly the same steps as in the proof of (1) of Theorem 2 , we can easily show that

$$
D_{n} \leq \frac{M_{1} M_{2}}{\alpha}\left(1-t_{n}\right)-\frac{M_{1} M_{2}}{\alpha}\left(1-t_{n}\right)^{\alpha+1} .
$$

Now since $(1-t) \in \ell$ is given and $A_{\alpha, t}$ is an $\ell-\ell$ matrix implies that $(1-t)^{\alpha+1}$ is in $\ell$ by [3, Thm. 1], it follows that $D \in \ell$. Also using the same techniques as in the proof of (2) of Theorem 2, we can easily show that $A_{\alpha, t} S_{x} \in \ell$ and hence the assertion follows.

COROLlary 3. Suppose that $\alpha>0, q>1$, and $t_{n}=1-(n+2)^{-q}$, then every $A_{\alpha, t}$ matrix is $\ell$-translative for each $A_{\alpha}$-summable sequence $x$ in $\ell\left(A_{\alpha, t}\right)$.

Proof. Since by [3, Thm. 5], $A_{\alpha, t}$ is an $\ell-\ell$ matrix, the corollary easily follows by Theorem 3.

EXAMPLE 1. Suppose that $x_{k}=(-1)^{k}$ and $(1-t) \in \ell$; then every $\ell-\ell A_{\alpha, t}$ matrix is $\ell$-translative for the sequence $x$. Note that $x \in c\left(A_{\alpha}\right) \cap \ell\left(A_{\alpha, t}\right)$. If $-1<\alpha \leq 0$, then every $\ell-\ell A_{\alpha, t}$ matrix is $\ell$-translative for the sequence $x$ by Theorem 2. If $\alpha>0$, then every $\ell-\ell A_{\alpha, t}$ matrix is $\ell$-translative for the sequence $x$ by Theorem 3 .

EXAMPLE 2. Suppose that $\alpha>0, q>1$, and $t_{n}=1-(n+2)^{-q}$. Then every $A_{\alpha, t}$ matrix is $\ell$-translative for the unbounded sequence $x$ defined by

$$
x_{k}=(-1)^{k} \frac{k+\alpha+1}{\alpha+1} \text {. }
$$

Since $x \in c\left(A_{\alpha}\right) \cap \ell\left(A_{\alpha, t}\right)$, the assertion follows by Corollary 3 .

ACKNOWLEDGEMENTS. I want to thank my wife Mrs. Tsehaye Dejene and my two daughters Samera Mulatu and Abyssinia Mulatu for their great cooperation during my work on this paper.

\section{REFERENCES}

[1] D. Borwein, On a scale of Abel-type summability methods, Proc. Cambridge Philos. Soc. 53 (1957), 318-322. MR 19,134f. Zbl 082.27602.

[2] J. A. Fridy, Abel transformations into $l^{1}$, Canad. Math. Bull. 25 (1982), no. 4, 421-427. MR 84d:40009. Zbl 494.40002.

[3] M Lemma, The Abel- type transformations into l, to appear on the Internat. J. Math. Math. Sci.

[4] M. Lemma, Logarithmic transformations into ${ }^{1}$, Rocky Mountain J. Math. 28 (1998), no. 1, 253-266. MR 99k:40004. Zbl 922.40007.

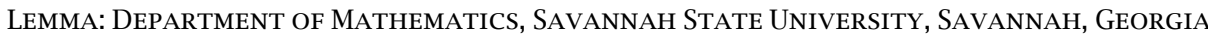
31404, USA 


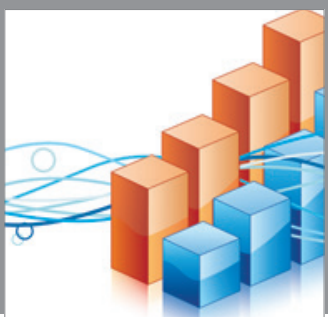

Advances in

Operations Research

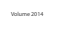

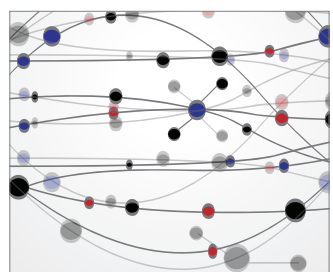

\section{The Scientific} World Journal
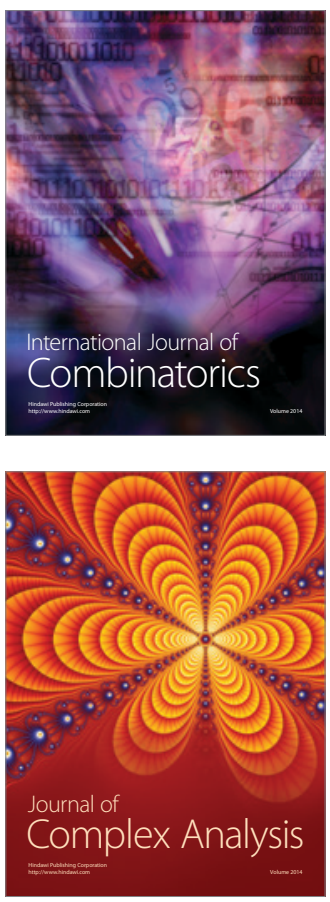

International Journal of

Mathematics and

Mathematical

Sciences
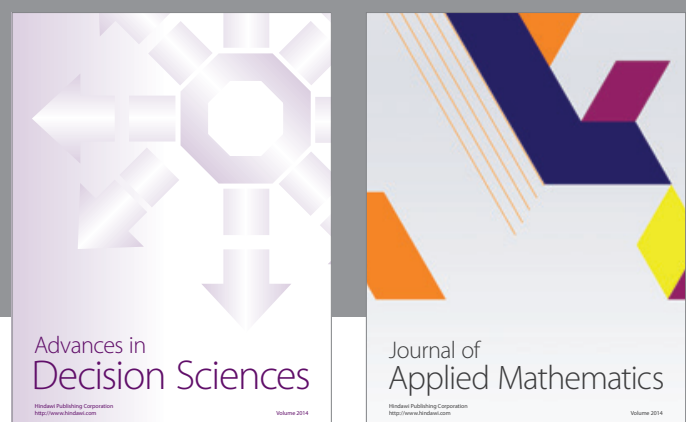

Journal of

Applied Mathematics
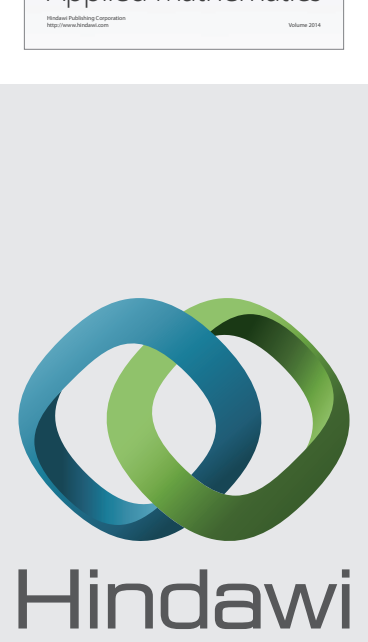

Submit your manuscripts at http://www.hindawi.com
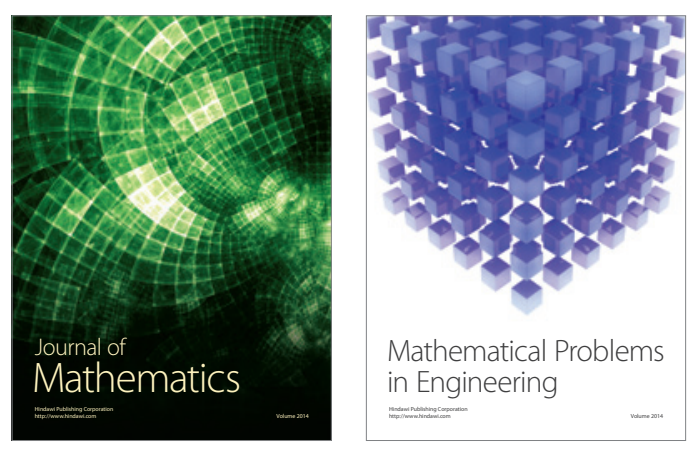

Mathematical Problems in Engineering
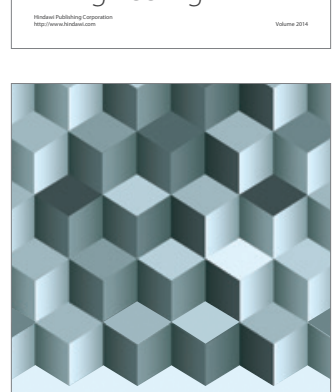

Journal of

Function Spaces
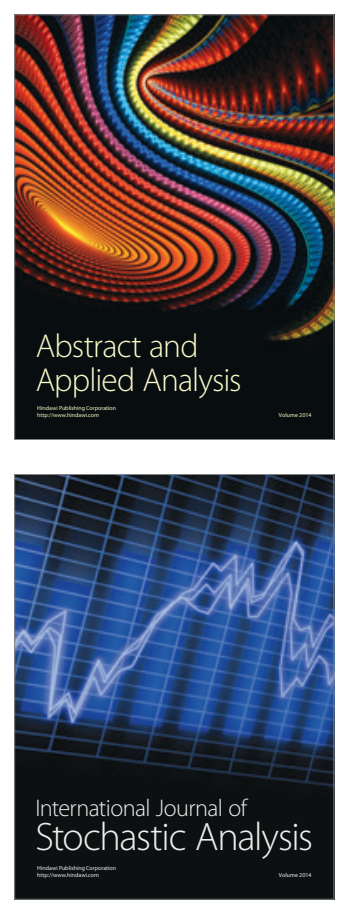

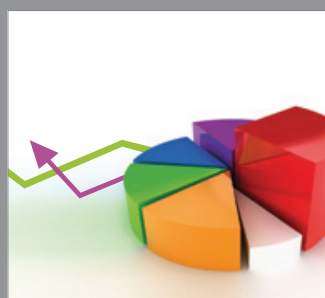

ournal of

Probability and Statistics

Promensencen
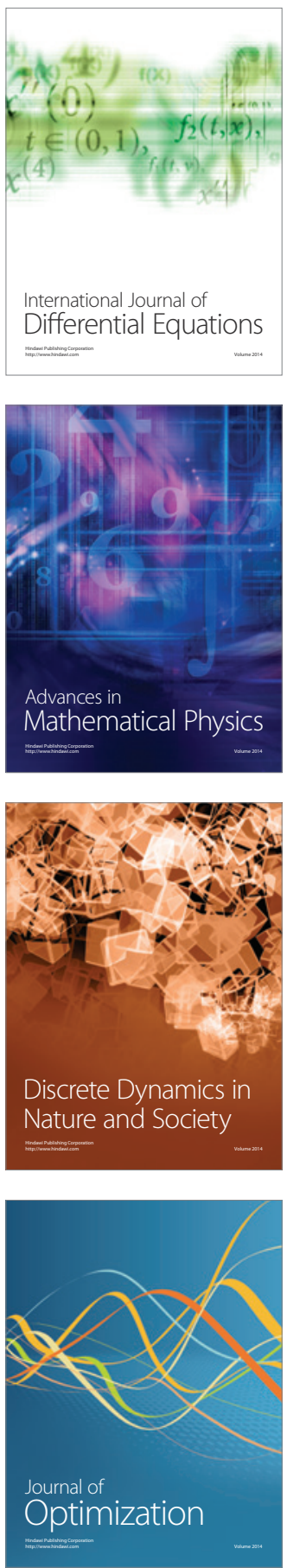